\title{
Multi-frequency pulsar studies at high radio frequencies
}

\author{
Michael Kramer \\ Max-Planck-Institut für Radioastronomie, Bonn, Germany
}

Kiriaki M. Xilouris

NAIC, Arecibo Observatory, Puerto Rico

\begin{abstract}
We report flux density measurements, polarimetric and timing observations of pulsars made at the highest radio frequencies to date, covering the widest frequency range from $1.4 \mathrm{GHz}$ to $86 \mathrm{GHz}$. We find that the magnetic field maintains its dipolar structure throughout the active part of the magnetosphere, a region located close to the stellar surface and confined to a small slab of a few stellar radii. The change in width and shape of pulse profiles saturates at $\mathrm{mm}$-wavelengths while the depolarization accelerates, leading to almost completely depolarized emission. Two pulsars seem to exhibit a turn-up in their flux density spectrum at $\mathrm{mm}$-wavelengths.
\end{abstract}

\section{Introduction}

It is generally believed that pulsar radio emission originates from a magnetospheric region confined by the last open field lines, whereas the actual emission altitude is thought to be a function of frequency (Cordes 1978). The frequency development of profile widths and component separation finds, in this frame, a natural interpretation since higher frequency emission originates from lower and hence narrower layers. In order to probe the deepest layers of the pulsar magnetosphere, we have conducted observations between $1 \mathrm{GHz}$ and $86 \mathrm{GHz}$. Using these results, we investigated pulse spectra, profile morphology and the magnetic field structure in a frequency range where radio signals are more immune to propagation effects.

\section{Spectra}

During the first detection of four pulsars at $\mathrm{mm}$-wavelengths, i.e. at $\lambda 8.84 \mathrm{~mm}$ $(34.8 \mathrm{GHz})$, we measured flux densities which were significantly larger than expected from extrapolations from lower frequencies (Wielebinski et al. 1993, cf. Malofeev et al. 1994). While for two pulsars we can explain these unexpected large flux densities by a moding behaviour $(\mathrm{B} 0329+54)$ or an inadequately known spectrum (B0355+54), data for B1929+10 and B2021+51 are only consistent with a flattening or even a turn-up in the spectrum (Kramer et al. 1996, cf. Fig. 1). These first results were confirmed by further observa- 

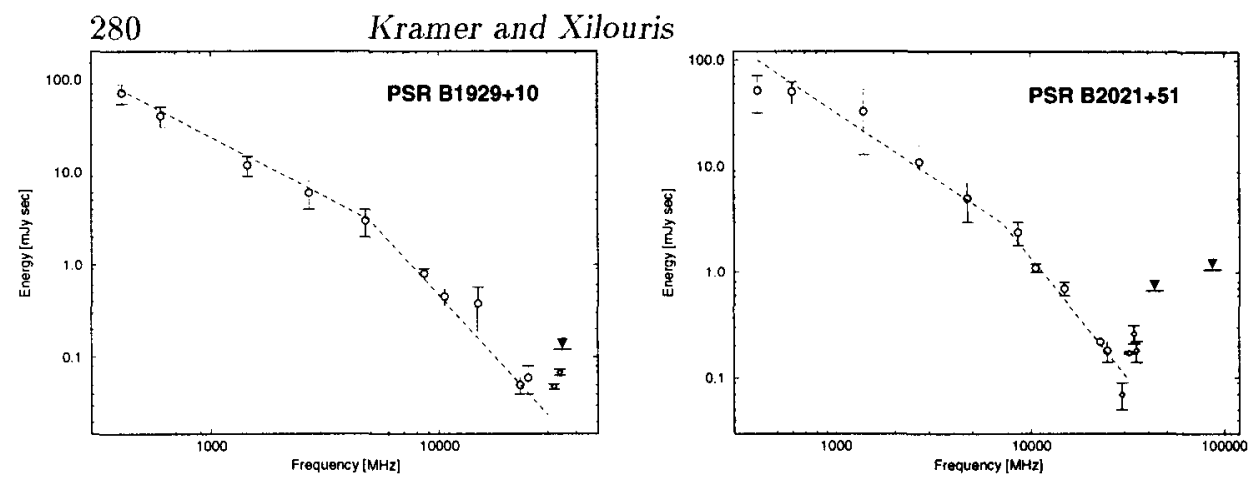

Figure 1. Flux density spectra for a) B1929+10 b) B2021+51. Upper limits for the $43 \mathrm{GHz}$ and $86 \mathrm{GHz}$ data are indicated.

tions which were done in a frequency range from $27.9 \mathrm{GHz}$ to $35.4 \mathrm{GHz}$. These observations also resulted in the detection of four additional pulsars where we determined flux densities consistent with values expected from lower frequencies, demonstrating the reliability of the calibration method applied (Kramer 1995). Attempts to confirm the observed spectral "turn-up" of B1929+10 and B2021 +51 by measurements at even higher frequencies, i.e. at $43 \mathrm{GHz}$ at Effelsberg (Kramer 1995) and at $86 \mathrm{GHz}$ with the $30 \mathrm{~m}$-dish of IRAM at Pico Veleta (Morris et al. 1995) were not successful due to weather conditions. In these cases only upper limits to the flux densities could be obtained.

\section{Polarization properties}

Polarization studies have been proven to be a very useful tool to investigate the underlying emission physics as well as to probe the magnetic field structure in the emission region (Radhakrishnan \& Cooke 1969). Therefore, we have also performed polarization measurements at the highest frequency ever, i.e. at 32 $\mathrm{GHz}$ (Xilouris et al. 1996). Most of the pulse profiles appear to be completely depolarized. Only B0355+54, and to some extent also B1929+10, show significant polarized emission. Investigating the frequency dependence, one finds that in most cases the degree of polarization remains fairly constant until a critical frequency, beyond which the polarization drops dramatically. For some pulsars like B1929+10 or B2020+28, this critical frequency is located in previously unaccessible regions ( $>15 \mathrm{GHz}$, cf. Fig. $2 \mathrm{a}$ ). In order to investigate the reason for this depolarization, we have traced the stability of the position angle. Although the range of longitudes with a defined position angle is smaller at high frequencies, a comparison of the angle swing at different frequencies shows that it remains stable, ensuring the persistence of an undisturbed dipolar field configuration (Xilouris et al. 1996).

\section{Emission heights}

Emission heights can be estimated from profile widths if the viewing geometry of the pulsar, i.e. the relative orientation of magnetic and spin axis and the observer's line-of-sight is known (e.g. Gil \& Kijak 1993). Assuming dipolar 

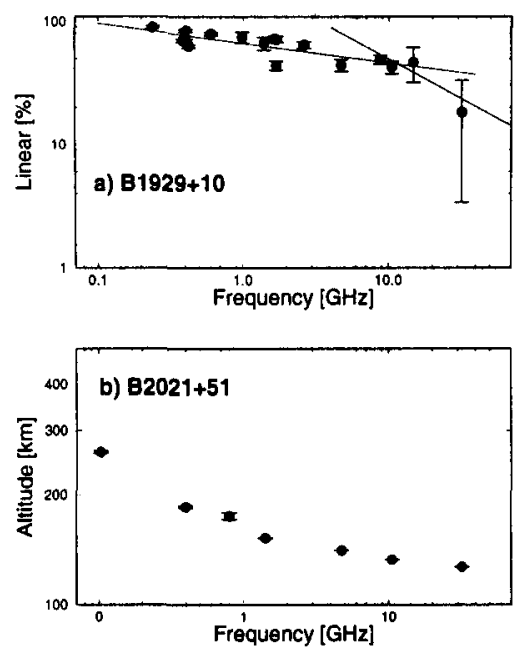

c) Рร₹ В $2070+78$

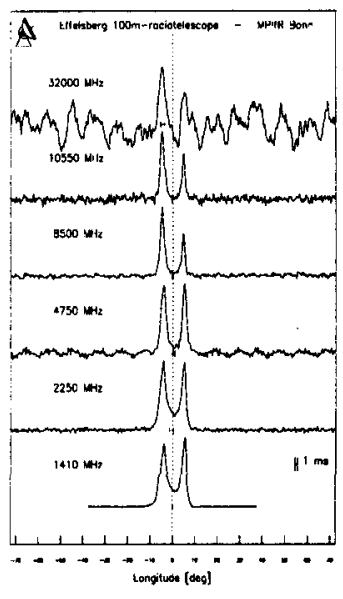

Figure 2. a) Degree of linear polarization vs. frequency for $\mathrm{B} 1929+10, \mathrm{~b})$ (geometrical) emission altitudes derived for $\mathrm{B} 2021+51$ and c) time-aligned profiles for B2020+28

field lines and (filled) circular emission beams, we used published geometry data (see Xilouris et al. 1996 and references therein) and the 10\%-intensity width of pulse profiles (taken from the literature and our own measurements) in order to determine emission heights at various frequencies. As a result we find that emission heights decrease from low to high frequencies and that they saturate to a value close to the pulsar surface at mm-wavelengths (cf. Fig. 2b). Using multi-frequency observations made between $1.41 \mathrm{GHz}$ and $32 \mathrm{GHz}$, we searched for effects caused by retardation, aberration and a sweepback of the magnetic field or higher order magnetic multipoles. Applying timing models kindly provided by the Jodrell Bank Group (Lyne, priv. comm.), we found a simultaneous arrival of the pulse profiles at all used frequencies, at least within the measurement accuracy (cf. Fig. 2c). This constrains the emission region to be less than $350 \mathrm{~km}$ in height, which is consistent with the results of the geometrical method described above, i.e. an upper limit of $550 \mathrm{~km}$ derived for a frequency range from $100 \mathrm{MHz}$ to $32 \mathrm{GHz}$.

\section{Discussion and Conclusions}

The measured spectra of B1929+10 and B2021+51 show a striking similarity to the theoretical spectrum for curvature emission of bunches where the bunch size determines the transition from coherent to incoherent radiation (Michel 1978). Thus, we investigated whether we observe incoherent curvature emission at high frequencies by calculating brightness temperatures $T_{B}$. We found that at 32 GHz $T_{B}$ drops by about $10^{7} \mathrm{~K}$ relative to $400 \mathrm{MHz}$. Although a calculation of the available coherence volume at $32 \mathrm{GHz}$ indicates that the observed luminosities ( $L \sim 10^{25} \mathrm{erg} / \mathrm{s}$ ) are too high to be explainable by (even coherent) curvature 
emission, other radiation properties indeed suggest that an increased fraction of incoherent emission is existing at high frequencies: Simple geometrical arguments cannot account for the observed depolarization especially since the effect accelerates towards frequencies where the geometry, as described by the pulse width and the inferred beam structure, remains stable. The observational data suggest that the active part of the magnetosphere is confined to a narrow region where a wide range of frequencies originates, making it improbable that propagation effects play an important role in the depolarization since the properties of the region are effectively the same for all those frequencies. The depolarization can, however, be explained by incoherent emission which would be less polarized than coherent emission. An increased occurrence of incoherent emission was already proposed by Bartel et al. (1980) in order to explain the increase of the pulse-to-pulse fluctuation above a critical frequency generally located at a few $\mathrm{GHz}$. Summarizing, the timing analysis, the smooth frequency development of profiles (cf. Fig. 2c) and the stable polarization angle argue against the existence of magnetic multipoles in the emission region of long period pulsars. While basic properties like the known moding behaviour of pulsars (Kramer et al. 1996) persist at high frequencies, some distinct changes take generally place at a few $\mathrm{GHz}$ : we often observe a break in the flux density spectrum (Malofeev et al. 1994), a break in the depolarization index, a saturation of the profile narrowing, an apparent break down of the radius-to-frequency mapping and a sudden increase in the pulse-to-pulse modulation index (Bartel et al. 1980). Concluding, we find new support for the old idea (cf. Cordes 1981) that radio emission changes significantly at a few $\mathrm{GHz}$, i.e. it becomes less coherent.

Acknowledgments. The authors are indebted to R. Wielebinski and A. Jessner for their inestimable help and support. It is also a pleasure to thank J. Gil, V. Malofeev and J.H. Seiradakis who were involved at early stages of this project. MK also wants to thank D. Morris and C. Thum for their invitation to participate at the Pico observations. Arecibo Observatory is operated by Cornell University under cooperative agreement with NSF.

\section{References}

Bartel, N., Sieber, W., Wolszczan, A. 1980, A\&A, 90, 58

Cordes, J.M. 1978, ApJ, 222, 1006

Cordes, J.M. 1981, in Pulsars, 13 years of Research on Neutron Stars, eds. Sieber W., Wielebinski R., (Dordrecht: Reidel), pp. 115-131

Gil, J.A., Kijak, J. 1993, A\&A, 273, 563

Kramer, M. 1995, PhD-thesis, University of Bonn

Kramer, M., Xilouris, K.M., Jessner, A., et al. 1996, A\&A, 306, 867

Malofeev, V.M., Gil, J.A., Jessner, A., et al. 1994, A\&A, 285, 201

Michel, F.C. 1978, ApJ, 229, 1101

Morris, D., Thum, C., Kramer, M. et al. 1995, IRAM, internal report

Radhakrishnan, V., Cooke, D.J. 1969, Astrophys. Lett., 3, 225

Wielebinski, R., Jessner, A., Kramer, M., Gil J.A., A\&A, 272, L13

Xilouris, K.M., Kramer, M., Jessner, A., et al. 1996, A\&A, in press 